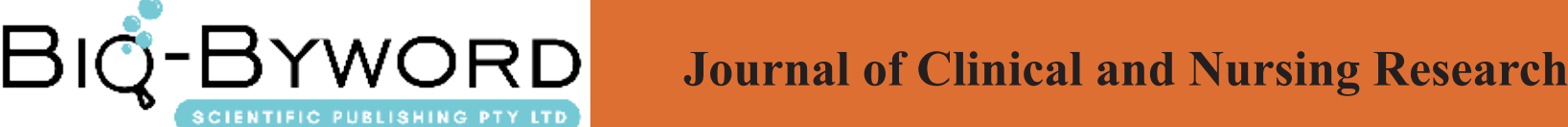

\section{The Application of Nursing Skills Training in the Implementation of Quality Nursing}

\author{
Zhang Xiaoyun, Li Wenyu, Yin Lijuan
}

People's Liberation Army No. 25 Hospital Outside

\section{ARTICLE INFO}

Article history:

Published online: 15th July, 2017

Key words:

Suffering from communication

skills training

quality care applications

\section{ABSTRACT}

Abstract: Objective: To explore the effect of nurse-patient communication skills training on patient satisfaction and nursing staff quality. Method: To develop nurse communication skills training programs, content and methods and implemented in 59 nursing staff, while constantly feedback information to be improved. Results: After training for nurse-patient communication skills, the patient's job satisfaction was improved and the comprehensive quality of the nurses was also improved. The data were compared before and after the differences were statistically significant $(\mathrm{P}<0.01)$. Conclusion: In the quality of nursing work through the training of nurses and patients with communication skills to achieve its objectives while improving the patient and nursing staff satisfaction and comprehensive quality is essential.

\section{Introduction}

Through the communication between nurses and patients in recent years, the relevant lifting methods continue to receive attention, so in the nursing process to further enhance their communication ability is very important, the following article through the choice of hospital nurses to communicate skills of the relevant training work. From the results point of view the effect is obvious, the relevant situation is reported as follows:

\section{Materials and methods}

\subsection{General information}

59 nurses were selected from May 2015 to June 2016 in the hospital, including deputy director of a nurse, 9 in charge of the nurse, also includes 21 nurses, in addition to 28 nurses. These nurses were distributed in the age of 20-48 years old, the average age distribution in the $(31.87 \pm 8.09)$ years old. The selected patients are mainly patients with ENT, the distribution of some of the more common diseases, the overall discharge of patients were evenly distributed (412.00 $\pm 13.00)$.

\subsection{Methods}

\subsubsection{Communication Skills Training:}


Development of training programs: first for the training of nurses and patients skills training to develop a general approach and plans through the practice of the program and program recycling. Nurses master the communication skills to enhance, so as to ensure that the patient's quality of service. Followed by the establishment of a clear communication development and skills. The contents of the nurse-patient included and the relevant matters to be clear through the development of the nurse-patient communication system to adopt for the quality control content to establish a special group to carry out the appropriate supervision. Improve the supervision and management system, so as to ensure the smooth completion of the communication system.

The main content of the training: in the process of training skills, contains a lot of content, first of all from the language training. In different occasions, for different patients to communicate in different languages, so whether from the admissions or in the nursing process. According to different circumstances according to different service language, so that patients can feel from the language of the enthusiasm of the nurses, the patient's psychology can play a very big comfort. Followed by intonation training in communication with patients. Remember not to be impatient and high tone. In the training if there is such a situation to be improved, in the tone as far as possible the use of suggestive and encouraging tone and then facial expressions training, in the face of patients to natural cordial, keep a smile of the demeanor, and finally is for nurse gestures and walking posture and other non-linguistic class training to enhance these communication skills, to achieve distance communication with the patient.

Training methods: In the training of nurse-patient communication skills and related knowledge, through the teaching of teachers in the communication of some linguistic or non-linguistic way of communication to explain such as how to properly use the ventilator, for some aphasia deaf patients how to communicate and how to communicate with patients who need tracheotomy, how to communicate and communicate with some younger or larger patients, and how to commu- nicate with the nurse's external image and civilization language and so play the importance of a clear, so as to enhance his cognitive ability. The second is through the training of teachers in person to demonstrate the case of the analysis, the students with the group.

\subsubsection{Evaluation methods}

Evaluation of patient satisfaction, through the training of nurses on the skills of training, the period is 1 year, and then through the hospital patient satisfaction survey form will participate in training before the situation and training after the completion of the case of comparative analysis, which will meet the degree of satisfaction is divided into three levels, there are very satisfied, satisfied and not satisfied with these three aspects. For the quality of nursing staff, mainly through the quality assessment and evaluation team to monitor the structure of the contents of the analysis, including assessment and assessment staff 5, the quality of each nurses to participate in training frequency points, using the percentage system (total score of 100 points), the main control of the head nurse manual and nursing staff for the monthly assessment of the structure of a comprehensive score for the nurses whether the use of labor Discipline, health education, standardized operation, etc. to communicate with each other, a total of 9, each 2 points for the points.

\subsection{Statistical methods}

The selected cases and related information through the technical information on the oath, the data through the $t$ test, the test level $\alpha=0.05$.

\section{Results}

Nursing skills training before and after the patient satisfaction and nurses' quality comparison, see Table 1.

\section{Discussion}

In the nursing work, to achieve the training of patients with communication skills in the patient during the entire hospital needs to be designed, and now China's

Table 1 Comparison of Patient Satisfaction and Nurses' Quality in Patients with Nursing Skills Training

\begin{tabular}{lllllll}
\hline Period & \multicolumn{4}{c}{ Patients' satisfaction } & \multicolumn{2}{c}{ Nurses' quality } \\
\cline { 2 - 7 } & Cases & Very satisfied & Satisfied & Not satisfied & People & Score $(\overline{\mathrm{x}} \pm \mathrm{s})$ \\
\hline Before training & 402 & 341 & 51 & 10 & 59 & $85.10 \pm 5.03$ \\
After training & 418 & 397 & 15 & 6 & 59 & $93.53 \pm 2.52$ \\
Statistical value & & & $\mathrm{Z}=4.792$ & & $t=12.033$ \\
$\mathrm{P}$ & & $<0.01$ & & $<0.01$ \\
\hline
\end{tabular}


medical development so rapidly, the competition between each other is increasing, high-quality nursing work to enhance hospital awareness, Therefore, it is very important to improve the nursing work in clinical practice. It is very important to realize the smooth language communication in nursing work. It is of great significance for patients and nurses. If there is any improper language, patients will bring a bad impression which will affect nurse-patient relationship. Satisfaction with communication skills training before and after comparison by this paper related research, data have found statistically significant $(\mathrm{P}<0.01)$. Thus, communication skills training is conducive to improving patient satisfaction, in order to enhance the hospital social image.

In summary, through the training of nurse-patient communication skills, nurses and patients can improve the enthusiasm of the teaching methods used in the relatively satisfactory, to improve the ability of nurse-patient com- munication is very helpful for the late to enhance the patient Satisfaction and better service for patients are of great significance, so training for nurse-patient communication skills can be better earned and worthy of promotion.

References:

[1] Wu Caiqin, Li Jingjing, Zhu Yunxia, Chen Fang. Nursing Skills Training in the Implementation of Quality Care in the Application [J]. Nursing Journal, 2012,07: 62-64.

[2] Yuan Xiaoling, Zhao Aiping. Clinical Low-Grade Nurses Nurse Communication Skills Training [J]. Nursing Journal, 2010,23: 51-53.

[3] Lei Rong, He Jian, Guan Ji, Long Lin, Li Quanqing, Zhao Qin. Nursing Skills Training To Improve the Clinical Communication Ability of Nursing Students [J]. Chinese Medical Equipment, 2013,11: 23-25。 